\title{
BMJ Open What works best when implementing a physical activity intervention for teenagers? Reflections from the ACTIVE Project: a qualitative study
}

\author{
Michaela James, ${ }^{1}$ Danielle Christian, ${ }^{2}$ Samantha Scott, ${ }^{1}$ Charlotte Todd, ${ }^{1}$ \\ Gareth Stratton, ${ }^{3}$ Joanne Demmler, ${ }^{1}$ Sarah McCoubrey, ${ }^{4}$ Julian Halcox, ${ }^{1}$ \\ Suzanne Audrey, ${ }^{5}$ Elizabeth A Ellins, ${ }^{6}$ Elizabeth Irvine, ${ }^{1}$ Sinead Brophy ${ }^{1}$
}

To cite: James M, Christian D, Scott S, et al. What works best when implementing a physical activity intervention for teenagers? Reflections from the ACTIVE Project: a qualitative study. BMJ Open 2019;9:e025618. doi:10.1136/ bmjopen-2018-025618

- Prepublication history and additional material for this paper are available online. To view these files, please visit the journal online (http://dx.doi. org/10.1136/bmjopen-2018025618).

Received 30 July 2018 Revised 28 March 2019 Accepted 5 April 2019

Check for updates

(c) Author(s) (or their employer(s)) 2019. Re-use permitted under CC BY-NC. No commercial re-use. See rights and permissions. Published by BMJ.

For numbered affiliations see end of article.

Correspondence to

Miss Michaela James;

m.l.james@swansea.ac.uk

\section{ABSTRACT}

Objective This paper explores what aspects of a multicomponent intervention were deemed strengths and weaknesses by teenagers and the local council when promoting physical activity to young people.

Design Qualitative findings at 12 months from a mixed method randomised control trial.

Methods Active Children Through Incentive VouchersEvaluation (ACTIVE) gave teenagers $£ 20$ of activity enabling vouchers every month for a year. Peer mentors were also trained and a support worker worked with teenagers to improve knowledge of what was available. Semistructured focus groups took place at 12 months to assess strengths and weaknesses of the intervention. Eight focus groups ( $n=64$ participants) took place with teenagers and one additional focus group was dedicated to the local council's sport development team $(n=8$ participants). Thematic analysis was used to analyse the data.

Results Teenagers used the vouchers on three main activities: trampolining, laser tag or the water park. These appeal to both genders, are social, fun and require no prior skill or training. Choice and financial support for teenagers in deprived areas was considered a strength by teenagers and the local council. Teenagers did not engage with a trained peer mentor but the support worker was considered helpful.

Conclusions The ACTIVE Project's delivery had both strengths and weakness that could be used to underpin future physical activity promotion. Future interventions should focus on improving access to low cost, fun, unstructured and social activities rather than structured organised exercise/sport. The lessons learnt from this project can help bridge the gap between what is promoted to teenagers and what they actually want from activity provision.

Trial registration number ISRCTN75594310

\section{BACKGROUND}

A notable decline in physical activity is seen in teenagers ${ }^{1}$ and many are insufficiently active. $^{2-4}$ Young people should participate in $60 \mathrm{~min}$ of moderate to vigorous activity a
Strengths and limitations of this study

- Study methodology is novel. Active Children Through Incentive Vouchers-Evaluation has been coproduced by young people to reflect their wants and needs.

- Used a semistructured focus group methodology to promote group interaction, which encourages indepth discussion.

> Only children consenting to take part in the study were able to be involved in the focus groups; these children could potentially be the more active and involved children, perhaps not capturing the views of those less engaged with activity.

- Only the council were consulted and the viewpoints of other stakeholders may have differed.

day $^{56}$; however, in Wales, only $11 \%$ of girls and $20 \%$ of boys meet this recommendation. ${ }^{7}$ The main barriers are reported to be cost and location, ${ }^{5-10}$ especially for teenagers from low socioeconomic backgrounds. ${ }^{11}$ Many interventions to improve activity in teenagers have chosen to focus on the school environment as it is considered a useful setting due to its ability to reach a large amount of teenagers. ${ }^{12} 13$ Previous interventions in this setting have been prescriptive, with specific activities or teaching strategies given to teenagers. ${ }^{4}$ 14-16 These interventions have had mixed success to date, often only increasing activity short-term ${ }^{15} 17$ as they fail to provide ongoing opportunities.

This style of intervention design and implementation is 'top down' with the emphasis on policymakers as the experts and sole designers. This results in a disconnect between what is provided and what teenagers actually need and want to do. ${ }^{810} 18$ Research shows that involving participants and those expected to deliver the intervention at an 
early stage aids the development of a strong intervention and increases the likelihood of success, effectiveness and sustainability. ${ }^{19} 20$ This approach has underpinned the Active Children Through Incentive Vouchers-Evaluation (ACTIVE) Project ${ }^{2}$ which was coproduced following discussions with teenagers regarding current activity provision in their area. ${ }^{18}$

The findings from this research confirmed that accessibility to activities was an issue. ${ }^{89}$ Teenagers wanted more opportunity to try new activities that were social and informal rather than more traditional forms of sport. In response to this, the ACTIVE randomised control trial aimed to empower teenagers to make their own activity choices via a voucher scheme, peer mentoring and support worker engagement. ${ }^{2}$ Multicomponent interventions of this nature are thought to be effective approaches to positively change physical activity. ${ }^{21}$ Financial incentives and peer mentoring have previously been shown to be beneficial to behaviour change. ${ }^{1722-24}$ A voucher-based intervention to increase activity in the UK has been previously tested in adults. ${ }^{25}{ }^{26}$ However, it remains uncertain whether a similar approach would be well received by teenagers.

The purpose of this paper is to explore what aspects of ACTIVE's multicomponent intervention (financial support, peer support, adult support) teenagers (the participants) and local council (intervention collaborators) perceived as the strengths and weaknesses when promoting physical activity in teenagers in deprived areas. It will also discuss what teenagers used the vouchers to do.

These findings can help inform the direction and implementation of future activity enabling interventions and policies for teenagers and young people.

\section{METHODS}

\section{Study design}

ACTIVE was a multicomponent intervention based in four secondary schools in Wales. It involved a voucher scheme, where all pupils in year 9 (aged 13-14 years) received £20 to spend on activity/equipment per month for 12 months (January to December) and incorporated peer mentoring and support worker engagement. Pupils selected the peer mentors (10 in each school) to act as 'champions' for physical activity in the school $^{27}$ (peer nomination questionnaire can be seen as online supplementary file 1 ). The support worker, who was a university employee, promoted the voucher scheme in the schools and provided a link between the schools and local council's sport development team. This was to promote collaborative working between the schools and the local council and to feedback any findings from ACTIVE or comments from teenagers. The project was funded by the British Heart Foundation ${ }^{28}$ and a detailed protocol of ACTIVE has been published. ${ }^{2}$ Consolidated criteria for reporting qualitative research guidelines were used to inform the analysis and presentation of this study. ${ }^{29}$

\section{Patient and public involvement}

ACTIVE was developed as a result of discussions with teenagers regarding activity provision and barriers to be active in their local community. ${ }^{2}$ Initial discussions resulted in a mixed method feasibility study of one school in a deprived area of Swansea, South Wales. ${ }^{8}$ This study was successful in improving fitness and physical activity ${ }^{8}$ and confirmed that teenagers found accessibility (eg, cost and lack of local facilities) as barriers. As a result, this trial was developed $^{2}$ alongside recommendations made by teenagers. The peer mentoring and support worker aspects were developed to provide ongoing involvement in the project for the teenagers. As findings emerged, ACTIVE reviewed them with the local council and other activity providers to align them better with what teenagers cared about and what they needed most. Findings from the study have been disseminated to participants and collaborators through conferences, social media and videos highlighting the key outcomes of the project.

\section{Outcomes}

This paper aims to present what activities teenagers' access when given the opportunity to do so in deprived areas. It also explores what aspects of a multicomponent intervention were deemed strengths and weaknesses when aiming to promote physical activity. Teenagers and the local council's sport development team were both included in this exploration to provide perspectives from those who the intervention targets and those who implement and have the power to change activity provision for this age group.

\section{Participants}

To be included in the study, schools needed to be located in one of Wales' Communities First catchment area. ${ }^{30}$ Four schools were recruited to the ACTIVE intervention (table 1). Following initial school recruitment, year 9 pupils (aged 13-14 years) gave consent to participate in the project's focus groups $(n=176)$, although all pupils in the year group received vouchers $(n=524)$. Participants were selected to be a part of focus groups purposively to ensure the views of those who had/had not engaged with the study were represented $(n=64)$. Pupils were considered engaged if they had spent more than the mean amount of vouchers $(\mathrm{n}=18$ vouchers $)$ used at the time the focus groups were carried out $(\mathrm{n}=73 / 176$ consented pupils). The focus groups also included at least one peer mentor. The local council sports development team were recruited via a monthly meeting between them and the project's support worker $(n=8)$. The total number of participants in the focus groups were 72 and 9 separate focus groups were conducted.

\section{Data collection}

Semistructured focus groups took place at the 12-month stage in the four intervention schools to assess the strengths and weaknesses of ACTIVE, if there were any recommendations and, whether it had made any 
Table 1 Demographics of schools

\begin{tabular}{lllll}
\hline & $\begin{array}{l}\text { Number of pupils in } \\
\text { year } \mathbf{9}(\mathbf{n}=\text { boys) }\end{array}$ & $\begin{array}{l}\text { Free school meal \% } \\
\text { in the school }\end{array}$ & $\begin{array}{l}\text { Welsh index of multiple } \\
\text { deprivation of the school* }\end{array}$ & $\begin{array}{l}\text { Mean vouchers used per } \\
\text { pupil at 12 months }\end{array}$ \\
\hline School 1 & $113(n=56)$ & 26.4 & 1660 & 17 \\
School 2 & $231(n=107)$ & 19.2 & 326 & 15 \\
School 3 & $125(n=59)$ & 10 & 84 & 17 \\
School 4 & $128(n=62)$ & 38.1 & 56 & 21 \\
\hline
\end{tabular}

*The higher the number the more deprived the local area.

difference to physical activity for teenagers. Focus groups were selected as the preferred methodology due to the promotion of group interaction, which encourages in-depth discussion. ${ }^{31}$ Eight focus groups lasting between 20 and $40 \mathrm{~min}$ took place, with boys and girls in separate groups to establish any gender differences. Members of the local council's sport development team also participated in an additional focus group to get an insight from the perspective of project collaborators and activity providers. The focus groups took place at the schools to ensure pupils remained in a familiar and convenient setting. The exception being the council focus group, which took place at the local council, chambers as part of a sports development team meeting.

A lead moderator facilitated the focus groups to ensure the discussion remained on the topic of interest. ${ }^{32} \mathrm{~A}$ topic guide which reflected the study's aims to ensure consistency across all focus groups and to provide triggers for discussion was used (online supplementary file 2). ${ }^{33}$ An assistant moderator was also present and was responsible for taking notes and audio recording. This role allowed key messages to be reported back to participants to ensure interpretation was correct and, to gain clarity over any points were unclear. This was a method of respondent validation. ${ }^{34}$ Both moderators had previously met participants during data collection and collaboration.

\section{Analysis}

The focus groups were transcribed in verbatim and names were removed to ensure anonymity. NVivo V.10 was used to manage and analyse the data. ${ }^{35}$ Two researchers separately analysed the data and compared coding/themes in order to guarantee no new codes/themes emerged and there were instances of the same theme to ensure data saturation. ${ }^{36}$ The researchers used thematic analysis (TA) to identify and report patterns in the focus groups. Braun and Clarke's Phases of $\mathrm{TA}^{37}$ underpinned the coding process. The themes can be seen as online supplementary file 3 .

\section{RESULTS}

A total of 18 codes were consolidated into three themes that discussed the project's strengths and weaknesses: (1) ability to choose own activities, (2) using external influences (eg, peer mentors and a support worker) and (3) the intervention's settings.

\section{Ability to choose own activities}

Teenagers discussed the ability to choose their own activities with the vouchers as a notable strength of ACTIVE. Table 2 shows what the teenagers chose to do with their vouchers. The vouchers were collected directly from the activity providers by the support worker. Notably, all choices were unstructured and informal activities. Trampolining accounted for almost half of the voucher usage $(49.1 \%)$, this was followed by laser tag $(11.46 \%)$ and the water park (slides and surfing) $(7.27 \%)$. Table 2 shows a detailed breakdown of how the vouchers were used. Teenagers spent the vouchers on unstructured and informal activities.

Both boys and girls used the trampoline parks frequently, one boy explained '... I think the most popular would be [trampoline park] and that's quite a multi-sexual sport then, isn't it?' (boy, focus group 7). The choice allowed boys and girls to participate in the same activities which one boy (focus group 3) believed had made girls more active. Girls acknowledged there were 'loads of things' (girl, focus group 4) they could do with the vouchers that were more chilled than typical activity provision on offer. Boys also agreed that activity had become fun. There were a lot of places young people did not realise would count as activity which they saw as a strength of the project as it had changed perceptions of activity for the teenagers.

As well as this, there was no longer a concern about money. One boy noted that young people would find paying for activities as a barrier but 'now you've got the vouchers to pay for it' (boy, focus group 5). There was an agreement among teenagers who the vouchers had helped improve socialisation for this reason. The vouchers gave them the choice of doing 'something in the nights' (girl, focus group 8), on 'Saturday afternoons and Sunday afternoons' (boy, focus group 3) or when you are 'on holiday' (girl, focus group 6). One girl (focus group 8) stated that by being able to use the vouchers in a social capacity had made her more confident to be active.

The local council agreed that giving teenagers a choice was a strength of ACTIVE. They liked that teenagers could decide where and how and considered the vouchers as more of a leisure pass where teenagers could go and enjoy activities with their friends. They also believed that the choice aspect improved the sustainability of ACTIVE's impact on physical activity as some teenagers found an 


\begin{tabular}{lrrrr}
\hline \multicolumn{6}{l}{ Table 2 Frequency of voucher use } \\
\hline Activity & Total & \multicolumn{1}{l}{$\%$} & Girls & Boys \\
\hline Trampolining & 3692 & 49.10 & 1914 & 1778 \\
Laser tag & 862 & 11.46 & 514 & 348 \\
Water park & 547 & 7.27 & 291 & 256 \\
\hline Football & 407 & 5.41 & 7 & 400 \\
\hline Fitness equipment & 368 & 4.89 & 207 & 161 \\
Cycling equipment & 361 & 4.80 & 76 & 285 \\
Gym membership & 357 & 4.75 & 182 & 175 \\
Gym pay and play & 211 & 2.81 & 134 & 77 \\
Football equipment & 122 & 1.62 & 10 & 112 \\
Skateboard equipment & 94 & 1.25 & 62 & 32 \\
Swimming equipment & 77 & 1.02 & 71 & 6 \\
\hline Foot golf & 69 & 0.92 & 19 & 50 \\
Martial arts equipment & 55 & 0.73 & 28 & 27 \\
\hline Parkour & 48 & 0.64 & 0 & 48 \\
\hline Swimming & 48 & 0.64 & 20 & 28 \\
\hline Miscellaneous & 37 & 0.49 & 36 & 1
\end{tabular}

equipment

\begin{tabular}{lrrrr}
$\begin{array}{l}\text { Equipment from Nash } \\
\text { Sport }\end{array}$ & 34 & 0.45 & 7 & 27 \\
Skateboarding & 23 & 0.31 & 0 & 23 \\
\hline Equipment for school & 19 & 0.25 & 0 & 19 \\
\hline Badminton & 13 & 0.17 & 5 & 8 \\
\hline Boxing equipment & 13 & 0.17 & 0 & 13 \\
\hline Tennis equipment & 13 & 0.17 & 0 & 13 \\
\hline Rock climbing & 10 & 0.13 & 10 & 0 \\
\hline Tennis & 8 & 0.11 & 0 & 8 \\
\hline Martial arts & 6 & 0.08 & 6 & 0 \\
\hline Gymnastics & 4 & 0.05 & 3 & 1 \\
\hline Court hire & 4 & 0.05 & 0 & 4 \\
\hline Paintballing & 4 & 0.05 & 4 & 0 \\
\hline Aqua aerobics & 3 & 0.04 & 0 & 3 \\
\hline Kickboxing & 3 & 0.04 & 0 & 3 \\
\hline Play area & 3 & 0.04 & 2 & 1 \\
\hline Zumba & 3 & 0.04 & 3 & 0 \\
\hline Aqua zumba & 2 & 0.03 & 2 & 0 \\
\hline
\end{tabular}

they did not feel the need to go to them. There were issues raised with the selection of peer mentors. They suggested that a teacher should select the peer mentors 'to look at who does most sports in the school' as a good role model (boy, focus group 3). One member of the local council suggested that pupils should put themselves forward and then there be a vote, but another felt that 'perhaps the people that put themselves forward might not be the people that you actually want' (council focus group).

The teenagers thought that presence of the support worker was beneficial as they created awareness of what was new or 'if anything had changed, which was really informative and nice' (boy, focus group 1). However, some pupils noted that the timings of the support worker were not ideal; in particular, they said morning assemblies are a time when they do not pay attention. The council focus group noted that the support worker was a difficult role as it had a variety of responsibilities from voucher distribution to activity promotion and drop-in sessions in schools. They perceived the support worker role to be a hard position and that the personality of the individual was the most important factor when considering who should fill it.

\section{The intervention's settings}

Most teenagers stated that there was very little within walking distance and that more activities should be put in the local community. However, the local council felt teenagers did not know all that was available and felt there could be a greater awareness created of community provision. One council member suggested that ACTIVE could have promoted the providers better in the schools. The project could have showed a video, for example, as this might capture the kids or activity providers should promote more of 'showing what they [the teenagers] would get if they went to see these providers' (council focus group). They believed the promotion was a weak aspect of ACTIVE.

There was a lot of discussion centred on physical activity lessons in school. Teenagers wanted more opportunities to be active through 'sports clubs at (lunch) dinner break and break' (boy, focus group 5) in school, for timings of activities to be lengthened, school kit for physical education (PE) lessons to be more lenient and more choice offered. The local council discussed teacher's involvement in ACTIVE, as they believed they had a pivotal role in the project's success. Some teachers were really proactive and 'really pushed the project' (council focus group) therefore, the intervention ran well. However, in other schools, 'there wasn't that many links between the PE department' (council focus group) which hindered delivery.

Using external influences to promote physical activity was a contested issue on ACTIVE. When asked about the peer mentoring scheme, most teenagers were unaware of it. The peer mentors themselves said they did not have anything to do, that they needed more 'recognition of who they were' (girl, focus group 8) or that the scheme would have benefit from 'a meeting, once a month, or something' (boy, focus group 7). Some pupils also said

\section{DISCUSSION}

This study identifies three key themes were identified that addressed the strengths and weaknesses of the delivery of the ACTIVE Project. While teenagers and the local 
council saw choice and support worker engagement as strengths of the project, there were issues explored around peer mentoring and ACTIVE's settings.

Young people felt the vouchers allowed them to overcome the barrier of cost as they were able to pay for activities. This is a significant strength of the project as it addressed the accessibility barrier. ${ }^{8-10}$ When empowered to choose activities they wanted, teenagers chose accessible, fun, activities that appealed to both genders, needed no prior skill and no prebooking. This suggests to get teenagers active there should be more of these types of activities promoted and made available. ${ }^{810} 18$ The informality of the activities promoted socialisation, meaning teenagers could meet up with their friends more outside of school. This was influential in giving teenage girls in particular, the confidence and encouragement to be more active. ${ }^{18}$ The local council also believed this choice would benefit the long-term success of ACTIVE as the teenagers could also buy equipment. From this intervention, it appears that choice and chance to speak about their activity preferences is a significant factor in a successful physical activity intervention.

Teenagers and the local council agreed that provision of sport was not the way forward but there needed to be more unstructured, informal opportunities. ${ }^{10}$ The use of the vouchers suggested they were used for a range of activities including days out, something to do with friends, ways to improve confidence and self-esteem and to buy equipment. Previous interventions have chosen to promote structured activity ${ }^{14} 15$ and this may be a contributing factor to the lack of long-term success of these interventions. ACTIVE highlights that what is currently provided is not what teenagers want to do. Despite the evidence of peer mentoring working in other health interventions in this age group, ${ }^{22} 27$ most pupils seemed to have little to no awareness of the peer mentors or they believed there was issues with their selection. It was important for teenagers who the mentors act as role models for activity but noted that those selected ended up being the most popular rather than the most active. Therefore, it is essential that correct characteristics be sought after when selecting peer mentors and that a more rigorous selection process be put in place rather than the use of peer nomination questionnaire. ${ }^{27}$ There is not a one size fits all approach to peer mentoring. However, given the participants wanted to be active with their friends in a social and fun environment, it is possible that the peer mentor approach of a mentor is too structured and an 'expert' peer is the wrong approach for motivating teenagers in deprived areas. The support worker was seen as helpful and an important link between pupils, schools and collaborative partners. However, more could be done to strengthen the impact this role had in terms of school visit timings. In future, it would be useful to involve pupils from the beginning to discuss how an external influence could most benefit them.

In terms of the interventions' setting, some pupils queried how much was actually available in their area suggesting that lack of local facilities and accessibility was a significant barrier for these teenagers. ${ }^{89} 38$ The local council argued that there was a lack of awareness and they suggested that the support worker's role could improve awareness. This does highlight one of the difficulties in the support worker role; should they empower teenagers to be able to access activities they want or promote activities that are available but perhaps ignored by the teenagers.

Pupils agreeing that there was too little time and emphasis placed on activity in school. Teenagers wanted more opportunities to be active during school time and a choice in what they would like to participate in. ${ }^{10}$ This is something future physical activity promoting interventions should take note of, as teenagers expressed a need for a wider choice of activity in school. The local council noted that the person taking responsibility as the contact in the school was vital in the delivery of an activity intervention and that buy-in from them would ensure success. This is important as the wrong lead in a school could hinder an intervention. Previous research has acknowledged this as well, noting that those in charge (eg, intervention leads and head teachers) need to be willing to allocate time to increase opportunities for teenagers to be active. ${ }^{39}$ A more standardised approach to school and teacher investment would be beneficial, for example, ensuring the PE department are in charge of the project's delivery. School buy-in and promoting the importance of teenage activity levels and health also underpins this. The school is where teenagers spend a significant amount of time and any successful activity intervention needs to engage and have buy in from the school. ${ }^{1420}$

\section{LIMITATIONS}

The use of focus groups enabled a more in-depth exploration of teenager's barriers to physical activity; only children consenting to take part in the study were able to be involved in the focus groups. These children could potentially be the more active and involved children, perhaps not capturing the views of those less engaged with activity and health and subsequently, the ACTIVE intervention. Only the local council were asked to participate in a focus group from the perspective of a collaborator and activity provider. The viewpoints of other stakeholders may have differed based on their voucher usage, promotion from ACTIVE and funding (eg, if they were a charity or privately funded).

\section{CONCLUSION}

The ACTIVE Project's delivery had both strengths and weaknesses that could be used to underpin future physical activity promotion. Providing teenagers a choice coupled with financial support in deprived areas was a strength of the ACTIVE. Teenagers reported to be able to do activities they wanted with their friends and changed their perceptions of physical activity. Thus, providing evidence 
that a voucher scheme works to get young people more active. ${ }^{25} 26$ Teenagers would like this choice translated into the school setting and into community provision. However, there is some tension between what teenagers believe is on offer in their local area and what the council believes can be access. The take home message from this study is that more collaboration needs to happen between teenagers, activity provision and policymakers to ensure their wants and needs are met. Further work is needed on how the intervention's strengths and weaknesses can underpin a larger scale project that can reach a bigger number of teenagers. This work highlights recommendations for future work in promoting activity among young people; namely improving access to fun, unstructured and social activities.

\section{Author affiliations}

${ }^{1}$ College of Medicine, Swansea University, Swansea, UK

${ }^{2}$ Department of Sport and Physical Activity, Edgehill University, Ormskirk, UK

${ }^{3}$ College of Engineering, Swansea University, Swansea, UK

${ }^{4}$ Active Young People Department, City and County of Swansea, Swansea, UK

${ }^{5}$ Population Health Sciences, University of Bristol, Bristol, UK

${ }^{6}$ Institute of Life Sciences, Swansea University, Swansea, UK

Contributors Swansea University designed the study and collected, analysed and interpreted the data. The university also wrote this study paper and made the decision to submit. MJ wrote the first draft of the paper and all authors provided critical input and revisions for all further drafts. SB wrote analysis and results section and provided critical input and revisions for all further drafts. MJ, CT, SS, El, JD and SB undertook data collection and data analysis. DC, CT, SB, GS, JH, SA, SM and EAE designed the study, aided in interpretation of findings and supervision of study quality. The corresponding author attests that all listed authors meet authorship criteria and that no others meeting the criteria have been omitted. All authors would like to thank the staff at the participating schools for their cooperation during the study and the year 9 pupils for their views and opinions as well as participation.

Funding This work was supported by the British Heart Foundation (PG/16/16/32057) who peer reviewed the protocol at the time of grant application but had no further involvement other than providing funding (grant number: $\mathrm{PG} / 16 / 16 / 32057)$.

Competing interests None declared.

Patient consent for publication Not required.

Ethics approval The College of Human and Health Science Ethics Committee granted ACTIVE ethical approval (Reference: 090516).

Provenance and peer review Not commissioned; externally peer reviewed.

Data sharing statement Data are available on reasonable request from the corresponding author (http://orcid.org/0000-0001-7047-0049). Additional information regarding the study protocol can be requested. The corresponding author has the right to grant on behalf of all authors and does grant on behalf of all authors, a worldwide licence to the publishers and its licensees in perpetuity, in all forms, formats and media (whether known now or created in the future), to (1) publish, reproduce, distribute, display and store the contribution; (2) translate the contribution into other languages, create adaptations, reprints, include within collections and create summaries, extracts and/or abstracts of the contribution; (3) create any other derivative work(s) based on the contribution; (4) to exploit all subsidiary rights in the contribution; (5) the inclusion of electronic links from the contribution to third party material where-ever it may be located and (6) licence any third party to do any or all of the above.

Open access This is an open access article distributed in accordance with the Creative Commons Attribution Non Commercial (CC BY-NC 4.0) license, which permits others to distribute, remix, adapt, build upon this work non-commercially, and license their derivative works on different terms, provided the original work is properly cited, appropriate credit is given, any changes made indicated, and the use is non-commercial. See: http://creativecommons.org/licenses/by-nc/4.0/.

\section{REFERENCES}

1. de la Haye K, Robins G, Mohr P, et al. How physical activity shapes, and is shaped by, adolescent friendships. Soc Sci Med 2011;73:719-28.

2. James M, Christian D, Scott $S$, et al. Active children through individual vouchers - evaluation (ACTIVE): protocol for a mixed method randomised control trial to increase physical activity levels in teenagers. BMC Public Health 2018;18:1-8.

3. Farooq MA, Parkinson KN, Adamson AJ, et al. Timing of the decline in physical activity in childhood and adolescence: Gateshead Millennium Cohort Study. Br J Sports Med 2018;52.

4. Sutherland R, Campbell E, Lubans DR, et al. A cluster randomised trial of a school-based intervention to prevent decline in adolescent physical activity levels: study protocol for the 'Physical Activity 4 Everyone' trial. BMC Public Health 2013;13:57-67.

5. Department of Health. Start Active, Stay Active A report on physical activity for health from the four home countries' Chief Medical Officer [Internet].. $2011 \mathrm{https} / /$ /www.gov.uk/government/uploads/system/ uploads/attachment_data/file/216370/dh_128210.pdf\%5Cnhttps:// www.gov.uk/government/uploads/system/uploads/attachment_data/ file/216370/dh_128210.pdf.

6. Rafferty R, Breslin G, Brennan D, et al. A systematic review of school-based physical activity interventions on children's wellbeing. Int Rev Sport Exerc Psychol 2016;9:215-30.

7. Welsh Government. Health Behaviour in School-aged Children Wale: Key findings. 2015

8. Christian D, Todd C, Hill R, et al. Active children through incentive vouchers - evaluation (ACTIVE): a mixed-method feasibility study. BMC Public Health 2016;16:890-903.

9. Charlton R, Gravenor MB, Rees A, et al. Factors associated with low fitness in adolescents--a mixed methods study. BMC Public Health 2014;14:764-77.

10. James M, Todd C, Scott S, et al. Teenage recommendations to improve physical activity for their age group: a qualitative study. BMC Public Health 2018;18:1-9.

11. Brockman R, Jago R, Fox KR, et al. "Get off the sofa and go and play": family and socioeconomic influences on the physical activity of 10-11 year old children. BMC Public Health 2009;9:253-60.

12. Kipping RR, Howe LD, Jago $R$, et al. Effect of intervention aimed at increasing physical activity, reducing sedentary behaviour, and increasing fruit and vegetable consumption in children: active for Life Year 5 (AFLY5) school based cluster randomised controlled trial. BMJ 2014;348:g3256-13.

13. Borde R, Smith JJ, Sutherland R, et al. Methodological considerations and impact of school- based interventions on objectively measured physical activity in adolescents : a systematic review and meta-analysis.. 2017;10:476-90.

14. Jago R, Edwards MJ, Sebire SJ, et al. Bristol girls dance project (BGDP): protocol for a cluster randomised controlled trial of an afterschool dance programme to increase physical activity among 11-12 year old girls. BMC Public Health 2013;13:1003-19.

15. Lawlor DA, Jago R, Noble SM, et al. The Active for Life Year 5 (AFLY5) school based cluster randomised controlled trial: study protocol for a randomized controlled trial. Trials 2011;12:181-204.

16. B Owen K, Smith J, Lubans DR, et al. Self-determined motivation and physical activity in children and adolescents: a systematic review and meta-analysis. Prev Med 2014;67:270-9.

17. Finkelstein EA, Tan YT, Malhotra R, et al. A cluster randomized controlled trial of an incentive-based outdoor physical activity program. J Pediatr 2013;163:167-72.

18. Brophy S, Crowley A, Mistry R, et al. Recommendations to improve physical activity among teenagers--a qualitative study with ethnic minority and European teenagers. BMC Public Health 2011;11:412-25.

19. Howie EK, Stevick ED. The "ins" and "outs" of physical activity policy implementation: inadequate capacity, inappropriate outcome measures, and insufficient funds. J Sch Health 2014;84:581-5.

20. van Sluijs EM, Kriemler S. Reflections on physical activity intervention research in young people - dos, don'ts, and critical thoughts. Int J Behav Nutr Phys Act 2016;13:25-31.

21. Owen MB, Curry WB, Kerner C, et al. The effectiveness of schoolbased physical activity interventions for adolescent girls: A systematic review and meta-analysis. Prev Med 2017;105:237-49.

22. Hollingworth W, Cohen D, Hawkins J, et al. Reducing smoking in adolescents: cost-effectiveness results from the cluster randomized ASSIST (A Stop Smoking In Schools Trial). Nicotine \& Tobacco Research 2012;14:161-8.

23. Patel MS, Volpp KG, Rosin R, et al. A randomized trial of social comparison feedback and financial incentives to increase physical activity. Am J Health Promot 2016;30:416-24. 
24. Shin DW, Yun JM, Shin JH, et al. Enhancing physical activity and reducing obesity through smartcare and financial incentives: a pilot randomized trial. Obesity 2017;25:302-10.

25. Moller AC, Buscemi J, McFadden HG, et al. Financial motivation undermines potential enjoyment in an intensive diet and activity intervention. J Behav Med 2014;37:819-27.

26. Harland J, White M, Drinkwater C, et al. The Newcastle exercise project: a randomised controlled trial of methods to promote physical activity in primary care. BMJ 1999;319:828-32.

27. Starkey F, Audrey S, Holliday J, et al. Identifying influential young people to undertake effective peer-led health promotion: the example of A Stop Smoking In Schools Trial (ASSIST). Health Educ Res 2009;24:977-88.

28. British Heart Foundation. We fight for every heartbeat - British Heart Foundation. 2017 https://www.bhf.org.uk/ (Cited 21 Apr 2017)

29. Tong A, Sainsbury P, Craig J. Consolidated criteria for reporting qualitative research (COREQ): a 32-item checklist for interviews and focus groups. Int J Qual Health Care 2007;19:349-57.

30. Welsh Government. Communities first area profile. 2016.

31. Brown A. Strategy A. Conducting focus groups with children and young people: strategies for success. J Res Nurs 2010;12:1-31.

32. Murray C. Peer led focus groups and young people. Child Soc 2006;20:273-86.
33. Hyde A, Howlett E, Brady D, et al. The focus group method: insights from focus group interviews on sexual health with adolescents. Soc Sci Med 2005;61:2588-99.

34. Triangulation TH. Respondent validation, and democratic participation in mixed methods research. J Mix Methods Res 2012;6:111-23.

35. Eyre ELJ, Duncan MJ, Birch SL, et al. Low socio-economic environmental determinants of children's physical activity in Coventry, UK: a qualitative study in parents. Prev Med Rep 2014;1:32-42.

36. Saunders B, Sim J, Kingstone T, et al. Saturation in qualitative research: exploring its conceptualization and operationalization. Qual Quant 2018;52:1-15.

37. Braun V, Clarke V. Using thematic analysis in psychology. Qual Res Psychol 2006;3:77-101

38. Brophy S, Rees A, Knox G, et al. Correction: child fitness and father's $\mathrm{BMI}$ are important factors in childhood obesity: a school based cross-sectional study. PLoS One 2012;7:1-7.

39. Beets MW, Okely A, Weaver RG, et al. The theory of expanded, extended, and enhanced opportunities for youth physical activity promotion. Int J Behav Nutr Phys Act 2016;13:1-15. 